\title{
| Evaluation of EUV resist performance below 20-nm CD using helium ion lithography
}

\section{Diederik Maasa, Emile van Veldhovena, Renée Pohlmanna, Anja van Langen-Suurlingb, Paul Alkemade Sander Wuister ${ }^{c}$, RIk Hoefnagels ${ }^{c}$, Coen Verspaget ${ }^{c}$, Jeroen Meessen ${ }^{c}$ and Timon Fliervoet ${ }^{c}$}

aTNO, P.O. Box 155, 2600 AD, Delft, The Netherlands

${ }^{b} K a v l i$ Institute of Nanoscience, Delft University of Technology, Lorentzweg1, Delft, The Netherlands

'ASML, De Run 6665, Veldhoven, The Netherlands

\section{innovation for life}

\section{Introduction}

For EUV lithography, development of high performance EUV resists is of key importance ${ }^{1}$. This development involves studies into resist sensitivity, resolving power and pattern uniformity ${ }^{2}$. We have used a subnanometer-sized $30 \mathrm{keV}$ helium ion beam to expose chemically amplified (CAR) EUV resists.

We aim to show that Scanning Helium Ion Beam Lithography ${ }^{3}$ (SHIBL) is a useful and economically attractive technique to prescreen novel EUV resists prior to their final performance evaluation in an EUV scanner.

Mechanism for EUV Chemically Amplified Resists

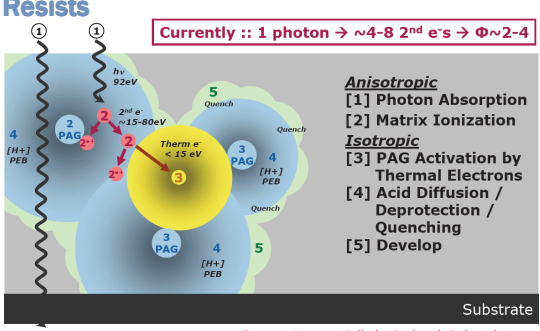

Figure 1 Info graphic on the activation mechanism of an EUV Chemically Amplified Resist. In SHIBL, step 1 and 2 are combined: the inelastic collision of a $30 \mathrm{keV} \mathrm{He}^{+}$ion with a resist atom generates low-energy secondary electrons directly. Figure by courtesy of T.R. Younkin (Intel).

EUV \& 30-keV He+ interaction in CAR resist There are remarkable similarities in the response of resists to $\mathrm{He}^{+}$ions and EUV photons: both create low-energy Secondary Electrons that activate the resist ${ }^{4-7}$ (see Fig. 1).

The main difference is that each $\mathrm{He}^{+}$ion has multiple inelastic collisions with the resist atoms, generating hundreds of SEs along its trajectory in the resist ${ }^{8}$, see Fig. 2.

The weak backscattering of the $\mathrm{He}^{+}$ions, (Fig. 2), results in ultra-low proximity effects. This enables the exposure of dense and detailed patterns by a focused $\mathrm{He}^{+}$ion beam without the need for proximity correction. Also EUVL capitalizes on low proximity, being it from a different origin.

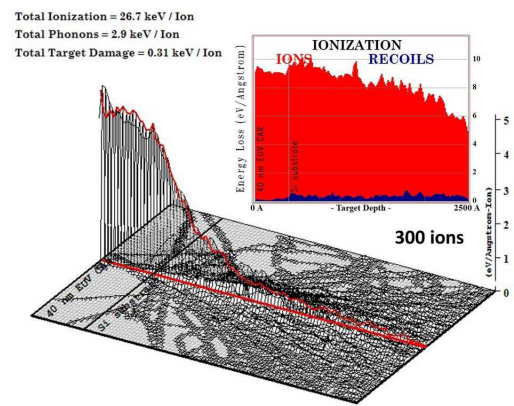

Figure 2 Energy loss of 30-keV $\mathrm{He}^{+}$ions through ionization of $40 \mathrm{~nm}$ thick EUV CAR resist on a Si substrate, computed by SRIM $^{8}$. The inset shows the energy loss through ionization (red) and recoil (blue) as a function of depth.

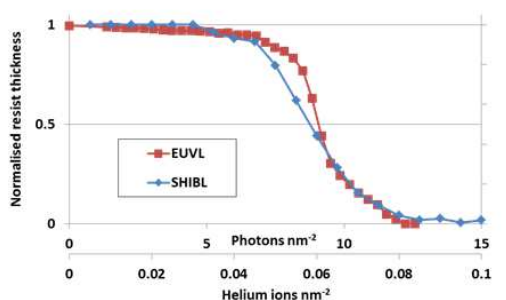

Figure 3 Large-area dose-response curves for EUV CAR resist of type A exposed to EUV and 30-keV $\mathrm{He}^{+}$. The EUV dose-to-clear is $18 \mathrm{~mJ} \mathrm{~cm}^{-2}$, the contrast is 3 . The $\mathrm{He}^{+}$ion dose-to-clear is $1.36 \mathrm{uC}$ $\mathrm{cm}^{-2}$ or $41 \mathrm{~mJ} \mathrm{~cm}^{-2}$, the contrast is 2 . Hence, compared to EUV photons, 150 times less $\mathrm{He}^{+}$ions are required for a same resist modification.

Methods

A $40 \mathrm{~nm}$ thick EUV CAR resist was exposed to 30 $\mathrm{keV} \mathrm{He}^{+}$ions in a Zeiss Orion Plus ${ }^{\mathrm{TM}}$ Helium Ion Microscope and to EUV in an ASML NXE:3300 scanner. Analysis was done using a Bruker Dektak NXT profilometer and a Hitachi CG-4000 CD SEM.

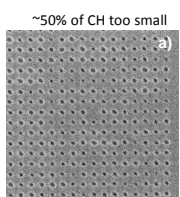

87 ions per $\mathrm{CH}$

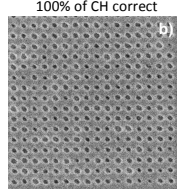

150 ions per $\mathrm{CH}$
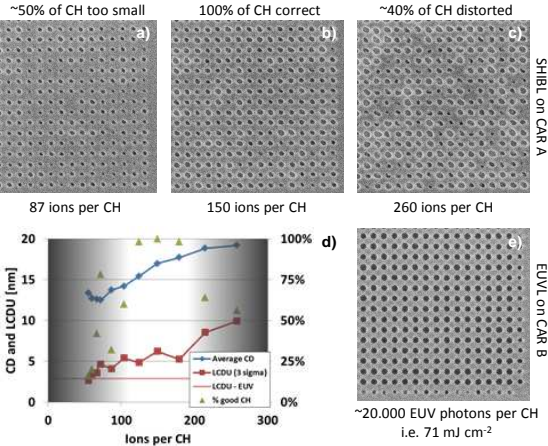

260 ions per $\mathrm{CH}$

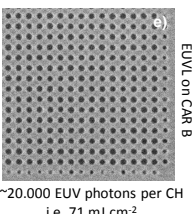

i.e. $71 \mathrm{~mJ} \mathrm{~cm}^{-2}$

Figure 4 CD SEM images of three dense arrays of point exposures, made in CAR resist of type A using SHIBL (a-c) and CAR resist of type $B$ using EUVL (e). The results of the CD analysis are shown in panel $d$. The ion dose ranged from $60-260$ ions per hole (in 9 panel d. The ion dose ranged from 60 - 260 ions per hole (in 9 steps with a dose-multiplication factor of 1.2), equal to 0.62 1.17 ion $\mathrm{nm}^{2}$ or $10-19 \mathrm{uC} \mathrm{cm}^{2}$. The EUVL contact hole CD $20.2 \mathrm{~nm}$. At low dose the smallest contact holes $(\mathrm{CHs})$ are rejected as closed, at high dose as distorted. Hence, only LCD values between $100-180$ ions per $\mathrm{CH}$ can be regarded as reliable.

Results and Discussion

The dose response curves from EUVL and SHIBL are rather similar, despite a different contrast (2 vs. 3). Per area, 150 times less ions than photons are needed for the same effect on the resist. $\mathrm{He}^{+}$ ions are estimated to generate 4 times more SEs per $\mathrm{nm}^{2}$ than the EUV.

Fig. 4a-c reveals considerable variations in the contact hole CD. However, the measured CD and LCDU (Fig. 4d) do not show strong variations, as many holes did not meet either the minimal-size or correct-shape criterion of the $C D$ evaluation $\mathrm{SW}^{9}$. Experiments at better conditions are necessary to draw final conclusions on SHIBL's hole printing capabilities.

Fig. 5 shows there is an ion dose that yields a 1:1 ratio of the line-and-spaces $C D$ for each pitch. At low dose some bridging is seen. The trench $C D$ and LWR are almost pitch-independent.

Fig 6. shows EUVL results from EUV resist B, with a highly comparable LWR as obtained with SHIBL.

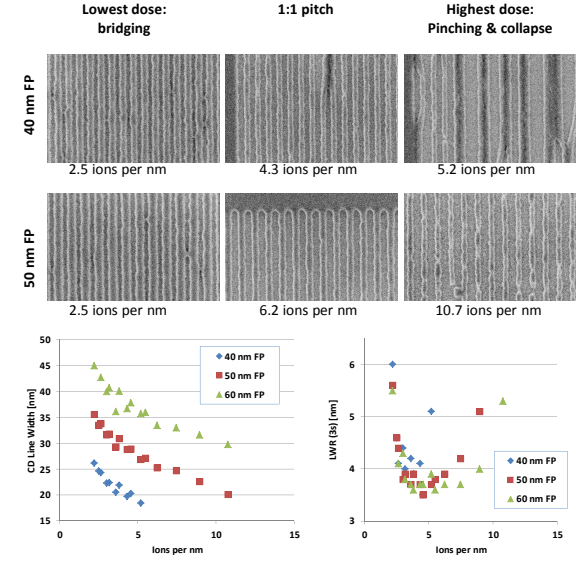

Figure 5 CD SEM images of lines-and-spaces at 40- and 50-nm pitch, exposed with SHIBL in CAR resist A at too low, optimal and too high dose. The graphs show line CD and LWR as a function of dose.
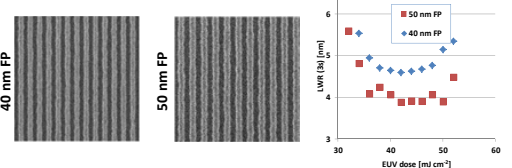

Figure 6 CD SEM image and LWR analysis of lines-and-spaces at 40 and 50-nm pitch, exposed with EUVL in CAR resist $B$ at optimal dose.

Conclusions

1. Scanning Helium Ion Beam Lithography is a promising novel method for the pre-screening of EUV resists on fundamental properties like resolution, sensitivity and pattern uniformity. EUVL and SHIBL both activate resist through low-energy secondary electrons. In SHIBL, these SEs are generated directly by the $\mathrm{He}^{+}$ions, enabling more insightful studies of EUV CAR activation by SEs.

2. Using SHIBL, contact holes and trenches at sub-20 $\mathrm{nm}$ resolution were exposed in CAR EUV resist. The results are alike EUVL exposures, although the obtained pattern uniformity is worse. Especially for the $\mathrm{CHs}$, this is at least partly attributed to suboptimal settings of the SHIBL expose tool.

3. Since SHIBL neither shows proximity effects nor requires a physical mask, the printability of more complex patterns can be evaluated, potentially enabling early device R\&D...

REFERENCES Virwani, H.D. Truong, N. Arellano, K. Petrillo, G.M. Wallraff, W.D. Hinsberg, Y. Hua, Fundamental study of extreme UV resist line edge roughness: Characterization, experiment, and modeling, J. Vac. Sci. Technol. B $3006 F 506$ (2012)

G.M. Gallatin, Resist blur and line edge roughness, Proc. SPIE $\mathbf{5 7 5 4}$ (2005) 38-52 政 Sci. Technol. B 27, L18 (2009)

Testro Thawa, Radiation Chemisty in Che

J. Thack Jay, J. Cameron, V. Jain, P. LaBearo S. Coley, O Ongayi, M. Wagner, A. Rachford and J. Biafore, Pursuit of lower Critical Dimensional Uniformity in EUV resists, J. Photopolym. Sci. Technol. 26 (2013) 605-610

6. T.H. Fedynyshyn, R.B. Goodman, A. Cabral, C. Tarrio, T.B. Lucatorto, Polymer photochemistry at the EUV wavelength, Proc. SPIE, 7639, 76390A (2010)

J. Torok, R. del Re, H. Herbol, S. Das, I. Bocharova, A. Paolucci, L. Ocola, C. Ventrice Jr., E. Lifshin, G. Denbeaux, R. Brainard, Secondary electrons in EUV lithography, J. Photopolym.Sci.Technol. 26 (2013) 625-634

James F. Ziegler, M.D. Ziegler, J.P. Biersack, SRIM - The stopping and range of ions ACKNOWLEDGEMENT

is research is supported by the Dutch Technology Foundation STW, which is part of Ne Netherlands Organization for Scientific Research (NWO), and which is partly funded by the Ministry of Economic Affairs. 